\title{
Dynamical behavior of a stochastic cholera model with differential infectivity and regime switching
}

\author{
qun liu ${ }^{1}$ and Daqing Jiang ${ }^{1}$
}

${ }^{1}$ Northeast Normal University

May 6, 2020

\begin{abstract}
In this paper, we study a stochastic cholera model with differential infectivity which is disturbed by both white noise and telegraph noise. Firstly, we prove that there exists a unique global positive solution to the system with any positive initial value. Then we obtain sufficient criteria for extinction of the diseases. Finally, we establish sufficient criteria for the existence and uniqueness of an ergodic stationary distribution of the positive solutions to the model by constructing a suitable stochastic Lyapunov function with regime switching. The stationary distribution implies that all the individuals can coexist and persist in the long term.
\end{abstract}

\section{Hosted file}

cholera models.pdf available at https://authorea.com/users/317572/articles/447629-dynamicalbehavior-of-a-stochastic-cholera-model-with-differential-infectivity-and-regime-

switching 